\title{
CERVICAL SPINE SIGNS AND SYMPTOMS: PERPETUATING RATHER THAN PREDISPOSING FACTORS FOR TEMPOROMANDIBULAR DISORDERS IN WOMEN
}

\author{
Débora BEVILAQUA-GROSSI ${ }^{1}$, Thaís Cristina CHAVES ${ }^{2}$, Anamaria Siriani de OLIVEIRA ${ }^{3}$
}

1- PT, Ph.D., Associate Professor, Department of Biomechanics, Medicine and Rehabilitation of the Locomotor Apparatus, Medical School of Ribeirão Preto, University of São Paulo, Ribeirão Preto, SP, Brazil.

2- PT, Ms degree in Health Sciences in Orthopedics, Traumatology and Rehabilitation, Department of Biomechanics, Medicine and Rehabilitation of the Locomotor Apparatus, Medical School of Ribeirão Preto, University of São Paulo, Ribeirão Preto, SP, Brazil.

3- PT, Ph.D., Professor, Department of Biomechanics, Medicine and Rehabilitation of the Locomotor Apparatus, Medical School of Ribeirão Preto, University of São Paulo, Ribeirão Preto, SP, Brazil.

Corresponding address: Débora Bevilaqua-Grossi - Departamento de Biomecânica, Medicina e Reabilitação do Aparelho Locomotor Faculdade de Medicina de Ribeirão Preto - Universidade de São Paulo - USP - Campus Universitário - 14049-900 Ribeirão Preto (SP) - Brazil Phone and fax: 551636024413 and 36330336 - e-mail: deborabg@fmrp.usp.br

Received: June 19, 2006 - Modification: December 04, 2006 - Accepted: March 09, 2007

\begin{abstract}
A

im: The purpose of this study was to assess in a sample of female community cases the relationship between the increase of percentage of cervical signs and symptoms and the severity of temporomandibular disorders (TMD) and vice-versa. Material and Methods: One hundred women (aged 18-26 years) clinically diagnosed with TMD signs and symptoms and cervical spine disorders were randomly selected from a sample of college students. Results: $43 \%$ of the volunteers demonstrated the same severity for TMD and cervical spine disorders (CSD). The increase in TMD signs and symptoms was accompanied by increase in CSD severity, except for pain during palpation of posterior temporal muscle, more frequently observed in the severe CSD group. However, increase in pain during cervical extension, sounds during cervical lateral flexion, and tenderness to palpation of upper fibers of trapezius and suboccipital muscles were observed in association with the progression of TMD severity. Conclusion: The increase in cervical symptomatology seems to accompany TMD severity; nonetheless, the inverse was not verified. Such results suggest that cervical spine signs and symptoms could be better recognized as perpetuating rather than predisposing factors for TMD.
\end{abstract}

Uniterms: Cervical spine disorders; Temporomandibular disorders; Perpetuating factors; Causality.

\section{INTRODUCTION}

Cervical spine disorders (CSD) are common conditions affecting the cervical region and related structures, with or without irradiating pain towards the shoulders, arms, interscapular region and head ${ }^{3,6,22}$. There are estimates that $67 \%$ of the population will suffer from neck pain at some stage of life ${ }^{10}$. Neck pain is often the major symptom in CSD related to post-traumatic or to chronic micro-traumatic lesions of the joints and periarticular structures ${ }^{3}$. Many studies have reported a higher percentage of CSD signs and symptoms in temporomandibular disorders (TMD) patients ${ }^{7,22,23}$, mainly when compared to non-TMD patients paired by age and gender ${ }^{21}$.

TMD are defined as the most common non-dental cause of orofacial pain ${ }^{17}$. TMD is a collective term applied to all problems related to the temporomandibular joint (TMJ) and associated musculoskeletal structures ${ }^{1}$. TMD characterizes a cluster of disorders marked by pain in the pre-auricular area, TMJ and masticatory muscles, as well as limitations or deviations during the mandible range of motion, and TMJ sounds during function ${ }^{8}$. Common patient complaints include headache, neck pain, face ache, and earache. Etiological factors are often divided into three categories: predisposing, perpetuating and precipitating ${ }^{17}$. In this context, CSD is considered as a predisposing and (or) perpetuating factor for TMD.

On one hand, if CSD is considered a predisposing factor for TMD, and supposing that the related orofacial pain ${ }^{2}$ is of cervical origin, there should be a direct relationship between the increase of TMD signs and symptoms and the previously existing CSD severity. Thus, cervical spine lesions caused by repetitive movements ${ }^{14}$, head and cervical posture alterations ${ }^{12,15}$, likely lead to CSD and, subsequently, to the manifestation of TMD signs and symptoms. On the other hand, if CSD is considered a perpetuating factor for 
TMD, and supposing that the referred neck pain ${ }^{2}$ is of orofacial origin, TMD signs and symptoms will not necessarily accompany CSD severity. In this approach, it is possible that TMD has its onset before CSD. Moreover, the further development of CSD signs and symptoms, would only contribute to TMD perpetuation. Several studies have reported a higher frequency of cervical spine signs and symptoms in TMD patients ${ }^{11,21,23}$. However, perhaps the inverse is not true, given that no significant differences were found between CSD patients and controls in terms of TMD signs and symptoms frequency ${ }^{6}$. Thus, it would be better to characterize CSD as a perpetuating rather than a predisposing factor.

Studies on chronic muscle-skeletal conditions are hampered by the fact that it is not possible to objectively diagnose the presence or absence of the disorder through tissue changes. Their recognition mainly relies upon the presence of isolated signs and symptoms of the disorders ${ }^{23}$. Anamnestic and clinical indices and scales are useful tools with clinical viability to assess the presence and severity of TMD and CSD signs and symptoms ${ }^{13,20,25}$.

Therefore, considering that CSD could function as either a predisposing or perpetuating factor for TMD, the aim of this study was to verify in a sample of adult women the existence of a relationship between the increase in cervical spine signs and symptoms and the progression of TMD severity. A secondary goal was to assess the opposite, i.e., associations between the increase of TMD signs and symptoms and its relation to the progression of CSD severity.

\section{MATERIALAND METHODS}

One-hundred women were randomly selected from a sample of college students to participate in this study. Participants were between 18 and 26 years old (21.43 \pm 1.80 years). The first 100 female volunteers that were submitted to cervical spine and TMD clinical examination and presented clinical signs and symptoms of TMD and CSD according to clinical indices of Helkimo ${ }^{13}$ (1974), Wallace and Klineberg ${ }^{25}$ (1993), respectively, were enrolled in this study. They were all community cases ${ }^{8}$ because none of them was under treatment for either of the conditions.

Exclusion criteria were the presence of general joint disorders involving the head and neck region (e.g.: rheumatoid arthritis), history of jaw fractures or orthognathic surgery, active TMD treatment, previous alterations of the cervical spine, and facial paralysis. The scientific and ethical aspects of the protocol were reviewed and approved by the local Ethics Committee and written informed consent was obtained from all participants.

The index of clinical craniomandibular dysfunction (ICCMD) and index of mandibular mobility (IMM), both proposed by Helkimo ${ }^{13}$ (1974), were used for assessment of TMD signs and symptoms. The ICCMD is composed of five items: limited mandibular range of motion, pain during mandibular range of motion, pain during TMJ and masticatory muscle palpation and during mandibular function.

Two indexes, similar to Helkimo's ${ }^{13}$, were applied to assess CSD signs and symptoms: the index of clinical craniocervical dysfunction (ICCD) and the index of cervical mobility $(\mathrm{ICM})^{25}$. The ICCD is composed of five items: cervical spine range of motion limitation, pain during cervical spine range of motion, alteration in cervical spine joints, pain on cervical muscles palpation and craniocervical posture. There are three possible scores $(0,1$ or 5 points) for each ICCMD and ICCD item, according to the answers obtained during clinical evaluation. Both indexes classify volunteers into four categories, according to disorder severity: without disorders (0 points), mild disorders (1-4 points), moderate disorders (5-9 points) and severe disorders (10-25 points).

A trained rater performed the manual palpation of masticatory (anterior, medial, and posterior fascicles of temporal and masseter muscles bilaterally) and cervical muscles (sternocleidomastoid, upper trapezius, and suboccipital muscles bilaterally) with a pressure of approximately 2 pounds for TMJ (lateral pole) and intraoral masticatory muscles (medial and lateral pterygoid muscles bilaterally) and 1 pound for the other structures?

IMM and ICM were administered to categorize the volunteers according to their severity of mandibular and cervical range of motion, respectively. The following mandibular movements were recorded: maximal mouth opening (MMO), maximal lateral deviation to right and left (MLDR and MLDE) and maximal protrusion (MP), according to Okeson ${ }^{19}$ (1998). The cervical movements of flexion, extension, right and left rotations and right and left lateral flexions according to Marques ${ }^{16}$ (2003). To measure mandibular $(\mathrm{mm})$ and cervical $\left({ }^{\circ}\right)$ range of motion, a boley gouge and a universal goniometer were used, respectively. The mean of three values of cervical and mandibular range of motion were obtained by a previously trained rater and were computed for statistical analysis.

Both IMM and ICM permit to classify volunteers into three categories of range of motion limitation: normal mobility ( 0 points), mild reduction in mobility (1-4 points), and severe reduction in mobility (5-20 points).

Descriptive statistics were performed to verify the percentages of volunteers in each severity category of TMD and CSD. Chi-square test $(\mathrm{p} \leq 0.05)$ was applied to analyze differences in the percentage of TMD and CSD signs and symptoms among severity groups. Differences among severity groups for mandibular and cervical range of motion were verified using one-way ANOVA $(\mathrm{p} \leq 0.05)$, as it was verified a normal distribution of the data according to the application of Shapiro-Wilk test $(\mathrm{p} \leq 0.05)$.

\section{RESULTS}

A significant number of volunteers were classified as moderate or severe TMD patients, while for CSD a significant number of volunteers were classified as severe (Table I). It was verified that the severity of TMD accompanied the severity of CSD and vice-versa because the number of 
volunteers with TMD increased proportionally with the severity of CSD and vice-versa. This pattern could be observed in the diagonal of Table I, as $4 \%$ of the volunteers were classified as having mild TMD and CSD, 7\% moderate TMD and CSD, and 32\% severe TMD and CSD. Thus, $43 \%$ of the participants had, concomitantly, the same classification of TMD and CSD severity (Table 1).

An analysis was performed on the distribution of volunteers with TMD signs and symptoms into categories of CSD severity (mild, moderate and severe) (Table II). There was a significantly lower percentage of severe CSD volunteers with tenderness to palpation of posterior fascicle of left temporal muscle, in relation to other severity categories (mild and moderate) (Table 2). Therefore, the increase in the severity of CSD signs and symptoms was not accompanied by an increase in the percentage of signs and symptoms of TMD (Table 2).

A significantly lower number of volunteers in the mild CSD group, compared to other severity categories, presented pain during maximum right lateral deviation of the mandible and in maximal protrusion, tenderness to palpation of right TMJ lateral pole and posterior pole of both TMJ, and tenderness to palpation of masseter and posterior fascicle of temporal muscle. Moreover, no differences were observed between moderate and severe CSD categories (Table 2), as well as regarding the mandibular range of motion in the categories of CSD severity ( $p>0.05$, ANOVA).

On the other hand, the analysis of the number of volunteers with TMD signs and symptoms distributed in the categories of CSD severity revealed that there was an association between TMD severity and increase in CSD severity (Table III). The percentage of pain during cervical extension, joint sounds during left lateral flexion and tenderness to palpation of the right upper trapezius and both suboccipital muscles were significantly greater in the severe TMD group than in the other categories of severity. The number of volunteers with pain during cervical flexion, joint sounds during rotation to right, tenderness to palpation of both sternocleidomastoid and left upper trapezius were significantly reduced only in the mild TMD group compared to the other severity categories (Table 3). Significant differences in the values of mandibular range of motion among TMD severity groups were not verified $(\mathrm{p}>0.05$, ANOVA).

\section{DISCUSSION}

The results of this study demonstrated that greater TMD severity implied greater CSD signs and symptoms. Thus, CSD signs and symptoms accompanied TMD severity. However the inverse was not verified, as TMD signs and symptoms were not more frequent in most CSD categories in women with TMD and CSD classified according to clinical evidences. Another important aspect was that, for $43 \%$ of the volunteers (Table 1), the classification of TMD and CSD severity was the same. However, in $57 \%$ of the cases, this trend was not observed. In this way, based on the results of this study, it could be suggested that CSD acts as a perpetuating factor and not necessarily predisposing factor for TMD.

These findings are in agreement with previous results ${ }^{21-}$ 23. Greater prevalence of cervical symptoms has been observed in patients with TMD, independently of the nature of the disorder (arthrogeneous, myogeneous or mixed disorders), when compared to healthy subjects ${ }^{23}$. StieschScholz, et al. ${ }^{21}$ (2003) reported that the incidence of "quiet CSD" was significantly higher in patients with symptoms of internal TMJ degeneration, compared to controls paired by gender and age. Quiet CDS has been previously described as the onset of signs of cervical disorders, which not necessarily leads to cervical symptoms.

De Wijer, et al. ${ }^{6}$ (1996) evaluated signs and symptoms of TMD in two groups of patients: one with major TMD complaints and another with major CSD complaints. The authors observed that the reported incidence of TMD signs and symptoms in patients with CSD was similar to that of healthy subjects. However, there are no studies investigating the association between TMD and CSD signs and symptoms in different severity categories considering a sample of volunteers simultaneously classified as having TMD and CSD.

Considering the cervical signs and symptoms that accompanied TMD severity, palpatory tenderness of cervical muscles was the most directly related symptom to this pattern of association. Stiesch-Scholz, et al. ${ }^{21}$ (2003) have also reported higher prevalence of cervical pain in patients with TMD than healthy subjects. However, considering that the present study sample consisted of TMD patients, it could be suggested that palpatory tenderness

TABLE 1- Percentage of volunteers distributed in the severity categories of Temporomandibular Disorders (TMD) and Cervical Spine Disorders (CSD)

\begin{tabular}{lccccc}
\hline & CSD Symptom-free & Mild CSD & Moderate CSD & Severe CSD & TMD (total) \\
\hline TMD Symptom-free & $0 \%$ & $0 \%$ & $0 \%$ & $0 \%$ & $0 \%$ \\
Mild TMD & $0 \%$ & $4 \%$ & $\%$ & $7 \%$ & $18 \%{ }^{*}$ \\
Moderate TMD & $0 \%$ & $4 \%$ & $7 \%$ & $24 \%$ & $35 \%$ \\
Severe TMD & $0 \%$ & $2 \%$ & $13 \%$ & $32 \%$ & $47 \%$ \\
CSD (total) & $0 \%$ & $10 \%$ & $27 \%$ & $63 \%{ }^{*}$ & $100 \%$ \\
\hline
\end{tabular}

${ }^{*} p<0.05$, chi-square test. 
of cervical muscles acts as a good predictive factor of TMD severity. Moreover, the classification in severity categories, made possible by the application of clinical indexes, could be an important tool for the characterization of patients with real treatment needs ${ }^{4}$ in samples of community cases.

Different from what was observed for palpatory tenderness of cervical muscles, mandibular and cervical range of motion did not accompanied the severity of CSD and TMD, respectively. This suggests that movement limitations of one system are not enough to distinguish the disorder severity of the other system. Previous studies have demonstrated that mandibular ${ }^{6}$ and cervical range of motion ${ }^{7}$ were not able to respectively differentiate patients with CSD and TMD.

Few temporomandibular signs and symptoms differentiated volunteers with moderate and severe CSD when compared to the number of cervical signs and symptoms that correctly distinguished volunteers with moderate and severe TMD. However, the analysis of the results demonstrated that volunteers classified as having moderate and severe CSD had an equally high incidence of temporomandibular signs and symptoms, which consequently contributed to the nonsignificant differences between these severity groups (Table 2). Such aspect

TABLE 2- Percentage of signs and symptoms of Temporomandibular Disorders (TMD) in the different severity categories of Cervical Spine Disorders (CSD) according to Wallace and Klineberg (1993)

\begin{tabular}{|c|c|c|c|c|}
\hline Signs and symptoms of TMD & $\begin{array}{l}\text { Total } \\
(n=100)\end{array}$ & $\begin{array}{l}\text { Mild CSD } \\
(n=10)\end{array}$ & $\begin{array}{l}\text { Moderate CSD } \\
(n=27)\end{array}$ & $\begin{array}{l}\text { Severe CSD } \\
(n=63)\end{array}$ \\
\hline \multicolumn{5}{|c|}{ Pain during mandibular movements } \\
\hline Mouth opening & $13 \%$ & $0 \%$ & $22.86 \%$ * & $10.64 \%$ \\
\hline \multicolumn{5}{|l|}{ Lateral deviation } \\
\hline Right & $9 \%$ & $0 \%$ * & $14.29 \%$ & $8.51 \%$ \\
\hline Left & $5 \%$ & $5.56 \%$ & $5.71 \%$ & $4.26 \%$ \\
\hline Protrusion & $12 \%$ & $0 \% *$ & $14.29 \%$ & $14.89 \%$ \\
\hline Joint sound during mouth opening & $23 \%$ & $16.67 \%$ & $20 \%$ & $27.66 \%$ \\
\hline \multicolumn{5}{|c|}{ Joint conditions } \\
\hline Blocked joint & $2 \%$ & $11.11 \% *$ & $0 \%$ & $0 \%$ \\
\hline Luxation & $5 \%$ & $11.11 \%$ * & $2.86 \%$ & $4.26 \%$ \\
\hline
\end{tabular}

Palpatory tenderness of masticatory structures

Masseter muscle

Right

Left

Anterior temporalis muscle

Right

Left

Posterior temporalis muscle

Right

Left

Medial pterygoid muscle

Right

Left

Lateral pterygoid muscle

Right

Left

TMJ - lateral pole

Right

Left

TMJ - posterior pole

Right

Left

${ }^{*} p<0.05$, chi-square test.
$42 \%$

$47 \%$

$27.78 \%$ * $\quad 40 \%$

$27.78 \%$ * $\quad 42.86 \%$

$34.29 \%$

$31.43 \%$

$17.14 \%$

$14.29 \%$

$20 \%$

$0 \%$

$45.71 \%$

$51 \%$

$38.89 \%$

$48.57 \%$

$59.57 \%$

$50 \%$

$44.44 \%$

$80 \%$

$70 \%$

$61.11 \%$

$62.86 \%$

$65.96 \%$

$67 \%$

$61.11 \%$

$37.14 \%$

$38.30 \%$

$34 \%$

$16.67 \%$ *

$45.71 \%$ *

$25.53 \%$

$68 \%$

$44.44 \%$ *

$71.43 \%$

$74.47 \%$

$61 \%$
$68.57 \%$ 
explains why the progression of CSD severity was not accompanied by a direct increase in temporomandibular signs and symptoms, as it suggests that more severe cervical spine disorders demonstrated similar frequencies of orofacial signs and symptoms.

Currently, a consensus has been reached about the real association between cervical and orofacial pain ${ }^{2}$. Hypotheses based on anatomical and structural aspects can be found in the literature to explain the coexistence of these disorders. Structural and neurophysiologic convergence of sensory information and inputs from the cervical muscles to nociceptive and not nociceptive neurons of the caudal subnucleus of the trigeminal nerve ${ }^{2}$, cervical branches $(\mathrm{C} 1$ the $\mathrm{C} 4)$ mainly related to the maintenance of head posture and inputs arriving at the trigeminal nucleus ${ }^{25}$, as well as, superficial sensory branches of upper cervical nerves supplying areas of the face as the mandibular angle ${ }^{5}$.
However, there is no agreement about the sequence in which the disorders appear when coexistence is verified. There is no consensus if it is a cervical disorder associated with a referred orofacial pain or an orofacial pain associated with a referred cervical disorder, or, yet, if a central nervous system modulation could deflagrate both disorders ${ }^{2}$. The results of this study demonstrated that cervical signs and symptoms tend to accompany TMD severity and that the inverse is not true. This suggests that, chronologically, TMD could appear before CSD. Another aspect that supports this hypothesis is that even volunteers with mild CSD presented a high incidence of orofacial signs and symptoms (Table 2). In one of the few studies reporting a possible orofacial origin for referred cervical pain ${ }^{18}$, it was observed that most TMD patients who received lidocaine into the TMJ presented relief of cervical pain.

On the other hand, the fact that the cervical signs and

TABLE 3- Percentage of cervical spine signs and symptoms in the different categories of Temporomandibular Disorders (TMD) according to Helkimo (1974)

\begin{tabular}{|c|c|c|c|c|}
\hline $\begin{array}{l}\text { Cervical spine signs and } \\
\text { symptoms }\end{array}$ & Total & $\begin{array}{l}\text { Mild TMD } \\
(n=18)\end{array}$ & $\begin{array}{l}\text { Moderate TMD } \\
(\mathrm{n}=35)\end{array}$ & $\begin{array}{l}\text { Severe TMD } \\
(n=47)\end{array}$ \\
\hline \multicolumn{5}{|c|}{ Pain in Cervical Movements } \\
\hline Flexion & $9 \%$ & $0 \% *$ & $11.11 \%$ & $9.52 \%$ \\
\hline Extension & $8 \%$ & $0 \%$ & $0 \%$ & $12.70 \%$ * \\
\hline \multicolumn{5}{|l|}{ Rotation } \\
\hline Right & $4 \%$ & $10 \%$ & $7.41 \%$ & $1.59 \%$ \\
\hline Left & $3 \%$ & $0 \%$ & $3.70 \%$ & $3.17 \%$ \\
\hline \multicolumn{5}{|l|}{ Lateral flexion } \\
\hline Right & $15 \%$ & $20 \%$ & $11.11 \%$ & $15.87 \%$ \\
\hline Left & $14 \%$ & $10 \%$ & $18.52 \%$ & $12.70 \%$ \\
\hline \multicolumn{5}{|c|}{ Joint Sounds } \\
\hline Flexion & $19 \%$ & $20 \%$ & $14.81 \%$ & $20.63 \%$ \\
\hline Extension & $12 \%$ & $10 \%$ & $14.81 \%$ & $11.11 \%$ \\
\hline \multicolumn{5}{|l|}{ Rotation } \\
\hline Right & $8 \%$ & $0 \% *$ & $3.70 \%$ & $11.11 \%$ \\
\hline Left & $8 \%$ & $10 \%$ & $0 \%$ & $11.11 \%$ \\
\hline \multicolumn{5}{|l|}{ Lateral flexion } \\
\hline Right & $13 \%$ & $10 \%$ & $11.11 \%$ & $14.29 \%$ \\
\hline Left & $16 \%$ & $10 \%$ & $3.70 \%$ & $22.22 \%$ * \\
\hline \multicolumn{5}{|c|}{ Palpatory tenderness } \\
\hline \multicolumn{5}{|c|}{ Sternocleidomastoid muscles } \\
\hline Right & $20 \%$ & $0 \% *$ & $14.81 \%$ & $25.40 \%$ \\
\hline Left & $17 \%$ & $0 \% *$ & $14.81 \%$ & $20.63 \%$ \\
\hline \multicolumn{5}{|l|}{ Trapezius muscle } \\
\hline Right & $83 \%$ & $40 \%$ & $70.37 \%$ & $95.24 \%{ }^{*}$ \\
\hline Left & $81 \%$ & $40 \% *$ & $74.07 \%$ & $90.48 \%$ \\
\hline \multicolumn{5}{|l|}{ Suboccipital muscles } \\
\hline Right & $71 \%$ & $40 \%$ & $48.15 \%$ & $85.71 \%$ * \\
\hline Left & $68 \%$ & $30 \%$ & $48.15 \%$ & $82.54 \%$ * \\
\hline
\end{tabular}

${ }^{*} p<0.05$, chi-square test. 
symptoms followed TMD severity does not necessarily indicate that TMD originates before CSD. In fact, CSD can originate before TMD and does not necessarily contribute to the development of signs and symptoms clinically detected, as the assessments performed in this study. Postural alterations, as, for example, forward head posture, could lead to symptoms of orofacial pain over time ${ }^{15}$. However, in the reviewed literature, there were no studies that verified the time required for development of orofacial pain signs and symptoms caused by head posture alterations.

\section{CONCLUSIONS}

The results of this study suggest that cervical signs and symptoms accompanied TMD severity. However, the inverse was not true, as TMD signs and symptoms did not increase with CSD severity in female community cases. These results suggest that TMD originates before CSD, and, therefore, cervical signs and symptoms could be better defined as perpetuating factors for TMD. Thus, the association between TMD and CSD is better defined as an orofacial disorder with referred cervical pain. However, it is not inevitably a cause-effect relationship, as CSD can originate before TMD and does not necessarily lead to the manifestation of signs and symptoms that could be detected by clinical assessment.

\section{REFERENCES}

1- American Society of Temporomandibular Joint Surgeons. Guidelines for diagnosis and management of disorders involving the temporomandibular joint and related musculoskeletal structures. Cranio. 2003;21(1):68-76.

2- Browne PA, Clark GT, Kuboki T, Adachi NY. Concurrent cervical and craniofacial pain: a review of empiric and basic science evidence. Oral Surg Oral Med Oral Pathol Oral Radiol Endod. 1998;86(6):63340

3- Ciancaglini R, Testa M, Radaelli G. Association of neck pain with symptoms of temporomandibular disorders in the general adult population. Scand J Rehabil Med. 1999;31(1):17-22.

4- Conti PC, Ferreira PM, Pegoraro LF, Conti JV, Salvador MC. A cross-sectional study of prevalence and etiology of signs and symptoms of temporomandibular disorders in high school and university students. J Orofac Pain. 1996;10(3):254-62

5- De Laat A, Meuleman H, Stevens A, Verbeke G. Correlation between cervical spine and temporomandibular disorders. Clin Oral Investig. 1998;2(2):54-7.

6- De Wijer A, Steenks MH, Bosman F, Helders PJ, Faber J. Symptoms of the stomatognathic system in temporomandibular and cervical spine disorders. J Oral Rehabil. 1996;23(11):733-41.

7 De Wijer A, Steenks MH, de Leeuw JR, Bosman F, Helders PJ. Symptoms of the cervical spine in temporomandibular and cervical spine disorders. J Oral Rehabil. 1996;23(11):742-50.
8- Dworkin SF, Huggins KH, Leresche L, Von Korff M, Howard J, Truelove E, et al. Epidemiology of signs and symptoms in temporomandibular disorders: clinical signs in cases and controls. J Am Dent Assoc. 1990;120:273-81.

9- Dworkin SF, LeResche L. Research diagnostic criteria for temporomandibular disorders: review, criteria, examinations and specifications, critique. J Craniomandib Disord. 1992;6(4):301-55.

10 - Falla D. Unravelling the complexity of muscle impairment in chronic neck pain. Man Ther. 2004;9(3):125-33.

11 - Fink M, Tschernitschek H, Stiesch-Scholz M. Asymptomatic cervical spine dysfunction (CSD) in patients with internal derangement of the temporomandibular joint. J Orofac Pain. 2002;20:192-7.

12- Gonzalez HE, Manns A. Forward head posture: its structural and functional influence on the stomatognathic system, a conceptual study. Cranio. 1996;14(1):71-80.

13-Helkimo M. Studies on function and dysfunction of the masticatory system. II. Index for anamnestic and clinical dysfunction and occlusal state. Swed Dent J. 1974;67(2):101-21.

14- Kirveskari P, Alanen P, Karskela V, Kaitaniemi P, Holtari M, Virtanen T, et al. Association of functional state of stomatognathic system with mobility of cervical spine and neck muscle tenderness. Acta Odontol Scand. 1988;46(5):281-6.

15-Mannheimer JS, Rosenthal R. Acute and chronic postural abnormalities as related to craniofacial pain and temporomandibular disorders. Dent Clin North Am. 1991;35:185-208.

16- Marques AP. Manual de goniometria. São Paulo: Manole; 2003.

17-McNeill C. Management of temporomandibular disorders: concepts and controversies. J Prosthet Dent. 1997;77(5):510-22.

18 - Norris CW, Eakins K. Head and neck pain: T-M joint syndrome. Laryngoscope.1974;84(9):1466-78.

19- Okeson JP. Management of temporomandibular disorders and occlusion. 3rd ed. Saint Louis: Mosby Year Book; 1998.

20 - Pehling J, Schiffman E, Look J, Shaefer J, Lenton P, Fricton J. Interexaminer reliability and clinical validity of the temporomandibular index: a new measure for temporomandibular disorders. J Orofac Pain. 2002;16(4):296-304.

21 - Stiesch-Scholz M, Fink M, Tschernitschek H. Comorbidity of internal derangement of the temporomandibular joint and silent dysfunction of the cervical spine. J Oral Rehabil. 2003;30:386-91.

22- Visscher CM, Lobbezoo F, Boer W, van der Zaag J, Verheij JG, Naeije M. Clinical tests in distinguishing between persons with or without craniomandibular or cervical spinal pain complaints. Eur J Oral Sci. 2000;108(6):475-83.

23- Visscher CM, Lobbezoo F, Boer W, van der Zaag J, Noeije M. Prevalence of cervical spine pain in craniomandibular pain patients. Eur J Oral Sci. 2001;109:76-80.

24- Visscher CM, Boer W, Lobbezoo F, Habets LL, Naeije M. Is there a relationship between head posture and craniomandibular pain? J Oral Rehabil. 2002;29(11):1030-6.

25-Wallace C, Klineberg IJ. Management of craniomandibular disorders. Part 1. A craniocervical dysfunction index. J Orofac Pain. 1993;7(1):83-8. 